\title{
When to include clinical study reports and regulatory documents in systematic reviews
}

\section{Tom Jefferson, 1,2 Peter Doshi, ${ }^{2,3}$ Isabelle Boutron, 4,5 Su Golder, ${ }^{6,7}$ Carl Heneghan, ${ }^{1,2}$ Alex Hodkinson, ${ }^{8,9}$ Mark Jones, ${ }^{2,10}$ Carol Lefebvre, ${ }^{11,12}$ Lesley A Stewart ${ }^{9,13}$}

10.1136/bmjebm-2018-110963

- Additional material is published online only. To view, please visit the journal online (http://dx.doi.org/ 10.1136/bmjebm-2018110963).

For numbered affiliations see end of article.

Correspondence to: Dr Tom Jefferson, Centre for Evidence Based Medicine, Nuffield Department of Primary Care Health Sciences, Primary Sciences Division, University of Oxford, Oxford OX2 6GG, UK; jefferson.tom@ gmail.com

\section{Abstract}

Reporting bias is a major threat to the validity and credibility of systematic reviews. This article outlines the rationale for accessing clinical study reports and other regulatory documents (regulatory data) as a means of addressing reporting bias and identifies factors that may help decide whether (or not) to include regulatory data in systematic reviews. The article also describes the origins and current state of regulatory data access and summarises a survey of current systematic reviewers' practices in considering regulatory data for inclusion in systematic reviews. How to access and extract regulatory data is not addressed. Organisations and other stakeholders such as Cochrane should encourage the use of data from clinical study reports as an important source of data in reviews of pharmaceutical interventions particularly when the intervention in question is of high importance and the risk of reporting bias is great.

\section{Introduction}

There has been a gradual realisation that sources of evidence historically considered to be reliable (such as peer-reviewed literature) are affected by reporting bias. Reporting bias generally refers to selective reporting of research depending on the nature and direction of research results. Reporting bias includes publication bias $^{1-3}$ and outcome reporting bias, ${ }^{1-5}$ among many others. ${ }^{6}$

Systematic reviews of randomised controlled trials play an important role in health decision-making. Most of these analyse data extracted from journal publications despite there being good evidence that reporting bias is widespread. As trials with unfavourable results are less likely to be published and unfavourable outcomes less likely to be reported within publications, the evidence base is often incomplete and skewed towards a positive spin. Systematic reviews that use only published data perpetuate such bias and possibly compound the issue through the credibility afforded by the systematic review, particularly if carried out by a trusted source such as Cochrane.

In a survey of 348 systematic reviews published in 2014, around three-quarters relied solely on data published in peer-reviewed journals. ${ }^{7}$ of those that accessed other sources, data from trials registries (such as ClinicalTrials.gov), conference proceedings or contacting authors were the most used. No reviews reported using or attempting to obtain regulatory information even though the majority of the reviews evaluated drug interventions. $^{7}$ A survey of 2184 Cochrane authors also found that contacting 'trialists/investigators' was one of the most common methods for accessing unpublished data and that data from manufacturers or from regulatory agencies were rarely obtained. $^{8}$

Clinical study reports (CSRs) are documents prepared and submitted to regulators to obtain a marketing licence for a pharmaceutical. They represent the most complete account of the planning, execution and results of such trials. CSRs contain some of the same information as journal papers (ie, rationale, objectives, methods, results, discussion/conclusion), but are substantially more detailed with numerous large tables and figures, and datasets not constrained by page limits. A CSR for a single trial may be hundreds, thousands or even tens of thousands of pages in length but they are generally relatively straightforward to navigate owing to their standardised and structured format. CSRs generally contain, as appendices, important study documents including the study protocol and any amendments, statistical analysis plan and any amendments, blank case report forms, patient information sheet, blank informed consent forms and individual patient listings. ${ }^{9}$

There are indications that CSRs may be incomplete, and in some cases, may be internally inconsistent between different sections of the same CSR. ${ }^{10}$ However, when comparing different data sources for the same trial, CSRs provide the greatest breadth and depth of information compared with journal articles, trial register data and grey literature. Aggregate data on subpopulations are often found in CSRs and can provide a source of further analysis. Such a wealth of information gives a fuller and more reliable picture of a trial's strengths and weaknesses, as well as a more reliable assessment of the benefits and harms of the studied interventions.

CSRs and other regulatory documents generally only exist for drugs and biologics. Non-pharmaceutical interventions (such as implantable devices, surgery, rehabilitation, behavioural (psychosocial) interventions and diagnostics) are responsible for a large part of healthcare expenditure and regulatory activity, but they do not generally produce CSRs. Transparency has generally 
been increasing in this area, although at a slower pace in the field of devices. Publicly funded trials, even of drugs and biologics, do not usually produce internationally standardised documentation, similar to a CSR.

In late 2010, the European Medicines Agency (EMA) began releasing CSRs of drugs and biologics on request under its Policy 0043. ${ }^{11}$ In October 2016, the EMA began to release CSRs prospectively under Policy 0070 (https://clinicaldata.ema.europa.eu). ${ }^{11} 12$ This policy applies only to documents received after 1 January 2015. Documents available from the EMA under Policy 0070 normally include the clinical overview, clinical summary and CSRs of individual trials. ${ }^{12}$ In 2017, Health Canada published a report announcing an initiative to publicly release clinical information concerning drugs and devices under an eventual EMA Policy 0070-like mechanism. ${ }^{13}$ In March 2018, the Food and Drug Administration (FDA) publicly released a CSR in a pilot programme that will eventually include nine new drug approvals. ${ }^{14}$ Some manufacturers are making CSRs available to reviewers (https://restoringtrials.org/insitutions-offering-data-access/). GlaxoSmithKline (GSK), for example, allows CSRs to be freely downloaded from its clinical study register, although the documents may be heavily redacted and incomplete. Other manufacturers are making CSRs available to researchers on request and after review and approval of their project proposal.

\section{Rationale for the consideration of regulatory documents (including CSRs) as sources of data for inclusion in systematic reviews}

Reporting biases can generally only be detected when two or more reports of the same trial are compared, for example, peer-reviewed publications compared with relevant regulatory documents. In addition to reporting bias, lack of transparency and lack of detail in journal publications may prevent or hinder detailed analyses of data which could be relevant to specific subpopulations potentially benefiting from or being harmed by the intervention. ${ }^{15}$ This situation is likely to be the consequence of compressing thousands of pages of text and tables into the historically restricted confines of a printed journal article. ${ }^{15}$

Table 1 contains a selected and illustrative list of studies that have compared different sources of data for the same trial, such as publication versus CSR or publication versus trial register entries. Although this is not an exhaustive list of all such studies, it covers $>50$ different interventions and offers insights into the ways in which reporting bias affects the biomedical literature.

The studies in table 1 strongly suggest that discrepancies in the reporting of trials across different sources of data are common. There are, however, limitations when interpreting discrepancies. First, different types of trial documents may have very different objectives. CSRs, for example, inform regulators and, by law, provide a comprehensive record of a study. Trials registers, in contrast, are primarily a visible collection of trial data, yet their reporting can be either absent or incomplete. Under some circumstances (such as for non-phase I trials of FDA-regulated drugs, devices or biologics), reporting of trials within ClinicalTrials.Gov, including the submission of results, is compulsory. ${ }^{16}$ There are also requirements for clinical trials funded by the US National Institutes of Health such as registration and reporting of results on ClinicalTrials.Gov, ${ }^{17}$ but these requirements are not always adhered to nor adequately policed. ${ }^{18}$

The generalisability of each finding of the studies in table 1 to the larger population of trials or topic areas that exist is debatable, and it is unclear whether reporting biases are lessening over time. Some journals have taken steps to limit the bias introduced by the current format of trial reporting, by requiring adherence to Consolidated Standards of Reporting Trials, ${ }^{19}$ by publishing the trial protocol, statistical analysis plan or supplementary data as an online appendix or by requiring data sharing as a condition of publication. ${ }^{20-22}$ As it is impossible to squeeze thousands of pages worth of information into a 10-page publication and the resulting information selection is based on unknown criteria, the authors of trial publications can, where these exist, provide links to the relevant CSR and other summary data.

We are currently aware of four systematic reviews (a Cochrane review of neuraminidase inhibitors, ${ }^{23}$ twin reviews of recombinant human bone morphogenetic protein 2 (rhBMP-2) ${ }^{24} 25$ and a review of reboxetine ${ }^{26}$ ) allowing an assessment of the contribution of regulatory data compared with the same trial data from published journal articles.

In the case of rhBMP-2, both CSRs and individual participant data (supplied separately by the manufacturer via the Yale Open Data Access Project) were included in the twin reviews, ${ }^{24} 25$ while the Cochrane review of neuraminidase inhibitors and the review of reboxetine were based on CSRs. ${ }^{23}{ }^{26}$ In all cases, the conclusions were that important aspects of the reviews were changed with access to the more complete data available in the CSRs. Access to the CSRs also provided a deeper understanding of the strengths and limitations of the trial evidence. In the case of the review of reboxetine, the inclusion of CSR data changed the conclusions of the review and allowed quantification of the exaggeration in favour of the effects of reboxetine compared with placebo and other SSRIs. ${ }^{26}$ The Cochrane review of neuraminidase inhibitors for influenza also found FDA drug-approval packages (medical and statistical officer reviews) to be an important source of data and detail.

As systematic reviews are considered as a gold standard of reliable research synthesis, we need to pay attention to the issue of reporting bias and to address whether, and how to decide when, accessing regulatory data, including CSRs, might offer a solution. The approach, however, is new and unfamiliar to most systematic reviewers and at the time of writing, regulatory data are not always immediately available. When available, using such documents can involve reviewing very large quantities of information, which may be time-consuming and resource intensive. Alternatively, it may be less time consuming than trying to assemble complete study data from information that is fragmented across several publications and unpublished sources such as trials registers. Thus, a framework to help identify where using data from regulatory documents is likely to matter most and prioritising those reviews which should adopt such an approach, will be helpful for groups grappling with how to respond to the increasing availability of these new sources of information.

\section{Current practice}

To raise awareness of the above issues and to assess the level of familiarity with and experiences of using data from CSRs and other regulatory documents within systematic reviews, we surveyed Cochrane and non-Cochrane authors to gauge how many had considered using regulatory data and how many had actually included such data in their reviews. There were 160 respondents with results mostly showing a lack of familiarity with regulatory sources of data, barriers to access and lack of resources to do so. The main rationale for authors seeking regulatory data, however, was minimisation of bias. $^{27}$ 


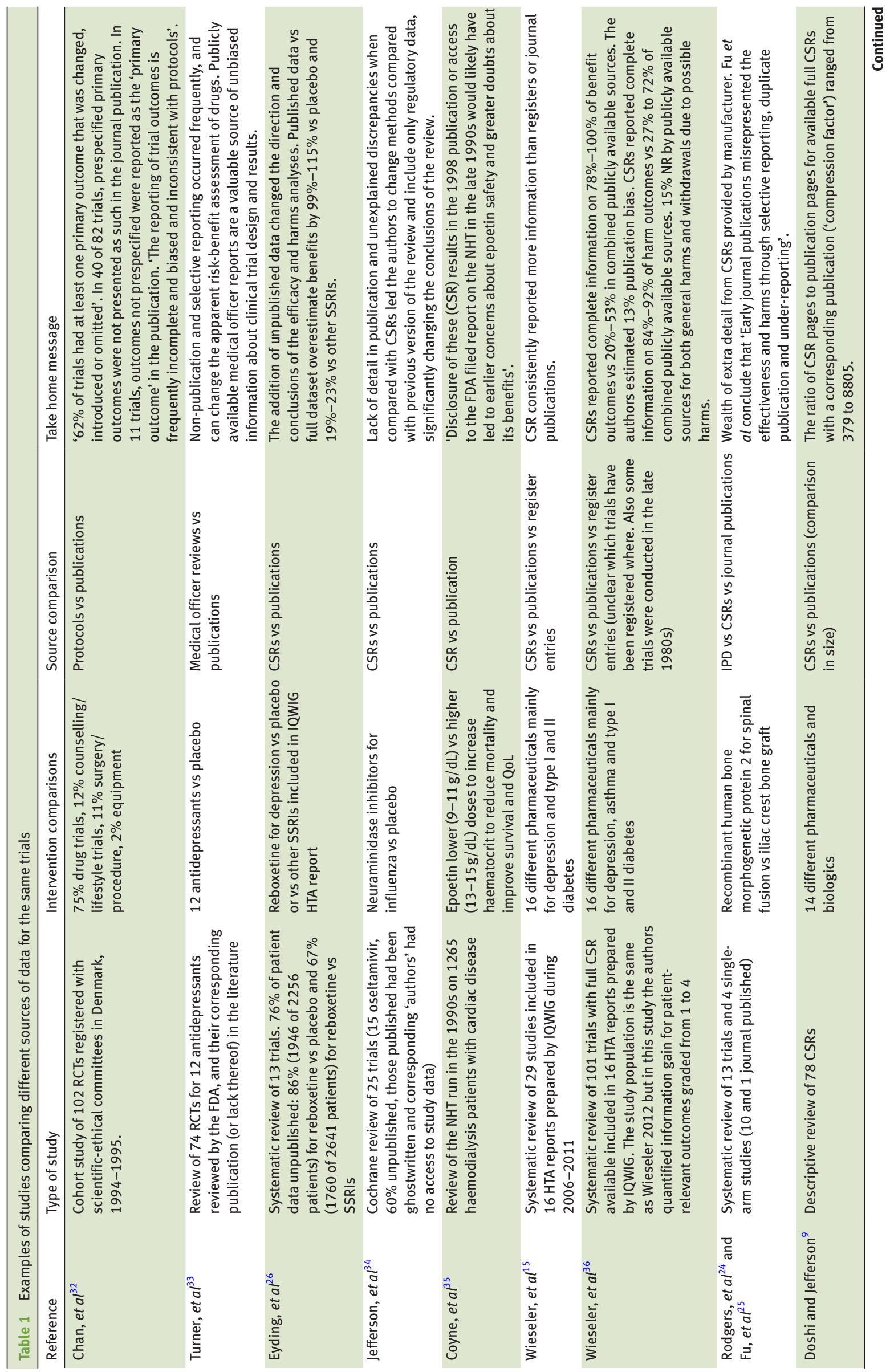




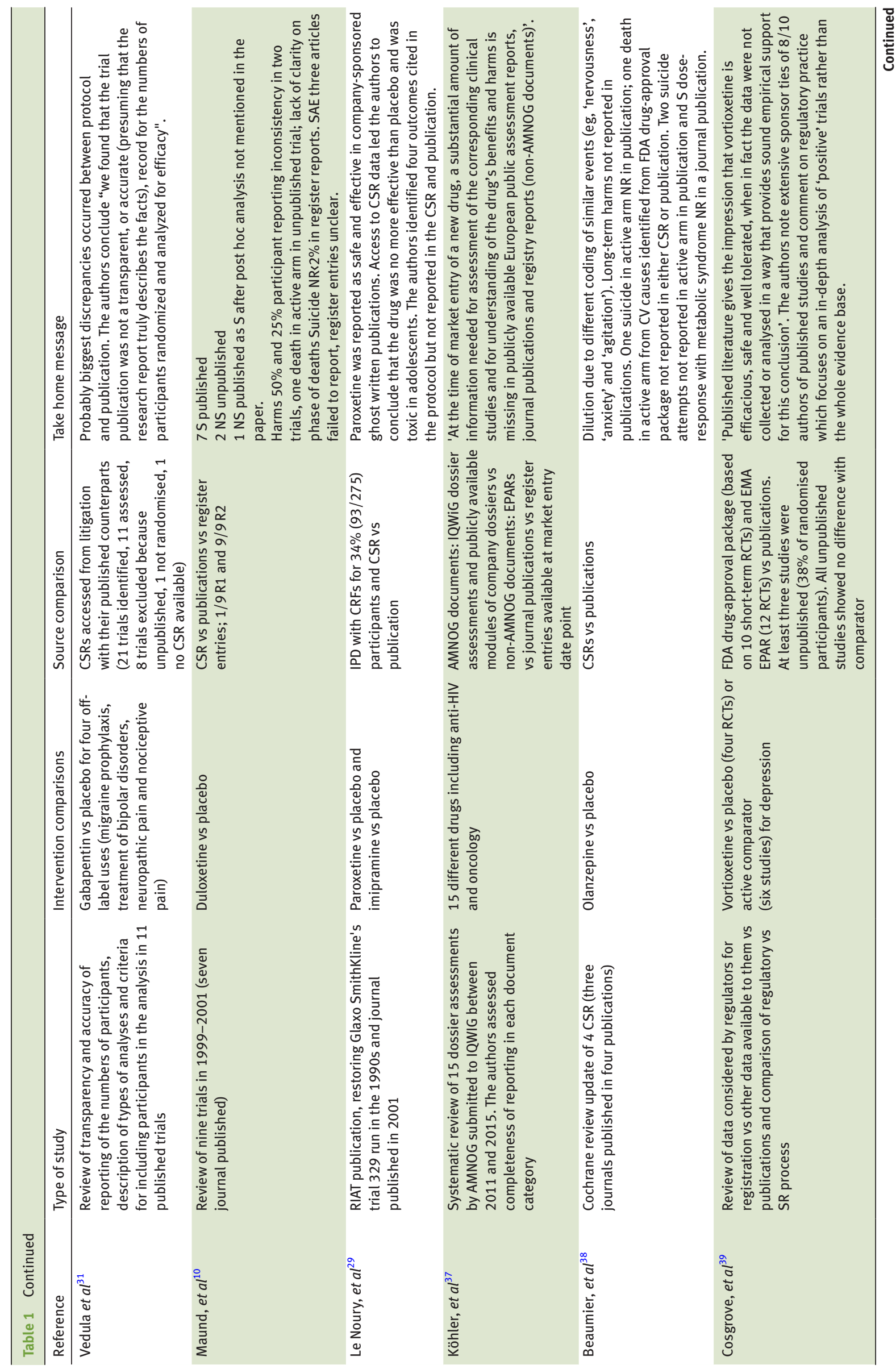

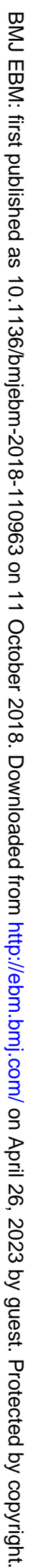




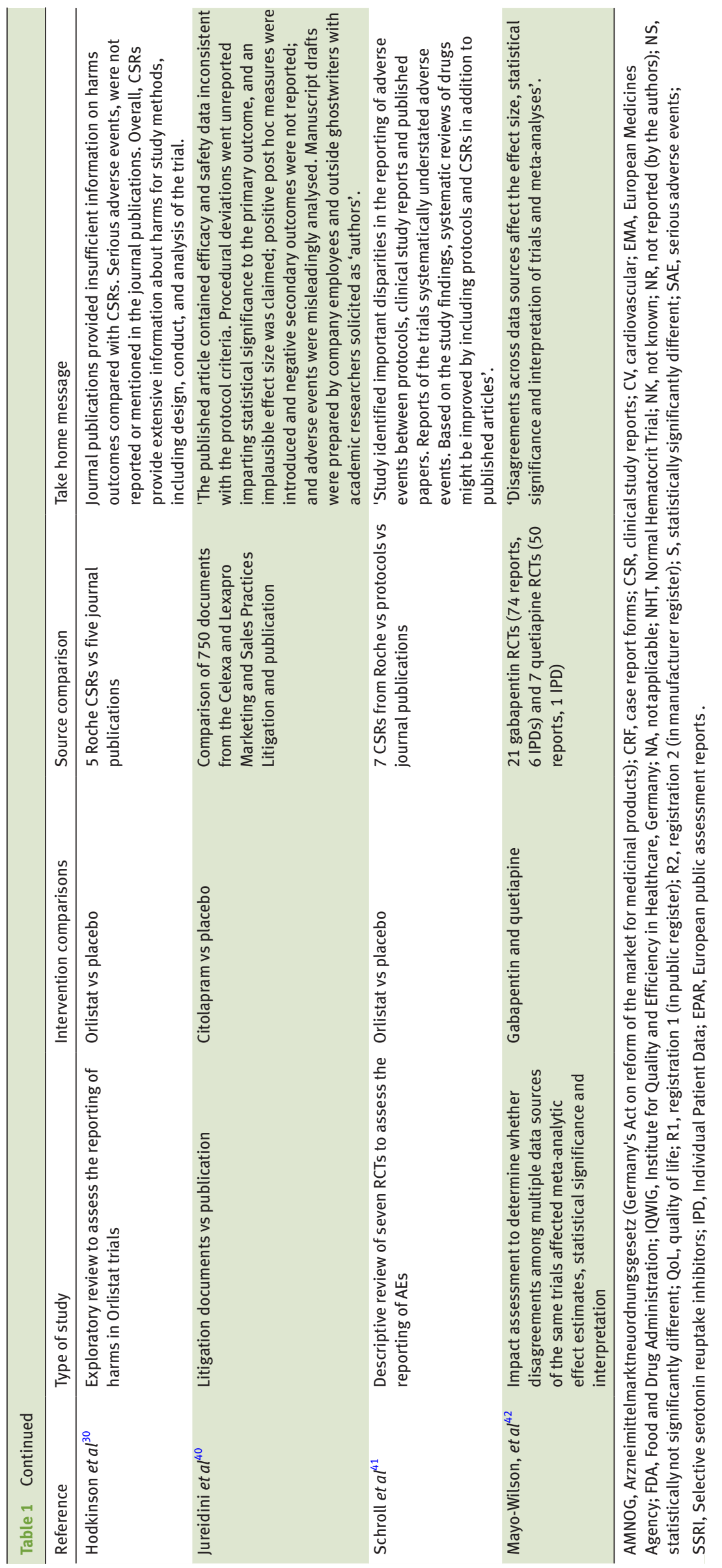

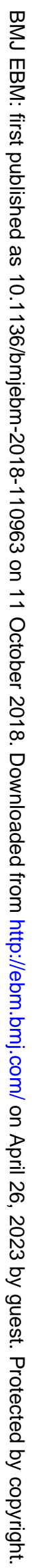


Table 2 Criteria for assessing whether to include regulatory data of a drug or biologic in a Cochrane review (not in order of priority)

\begin{tabular}{|c|c|}
\hline Criteria & Description of criteria \\
\hline 1 & $\begin{array}{l}\text { Monetary cost of the intervention on the healthcare budget } \\
\text { (ie, considering both the price of a course and the number of } \\
\text { people in the population that are being-or will be treated) }\end{array}$ \\
\hline 2 & $\begin{array}{l}\text { Burden of disease of the indication this product is meant to } \\
\text { treat/prevent }\end{array}$ \\
\hline 3 & Number of people using or likely to use the product \\
\hline 4 & Product new to the market \\
\hline 5 & $\begin{array}{l}\text { Product from a new drug class or has a new mechanism of } \\
\text { action }\end{array}$ \\
\hline 6 & $\begin{array}{l}\text { Has important interactions with other drugs (eg, drug-drug } \\
\text { interactions) }\end{array}$ \\
\hline 7 & $\begin{array}{l}\text { High proportion of randomised controlled trials evaluating } \\
\text { this product are industry funded }\end{array}$ \\
\hline 8 & $\begin{array}{l}\text { Prominent claims of safety and/or efficacy advantage of this } \\
\text { product over currently available treatments }\end{array}$ \\
\hline 9 & High degree of media attention surrounding this product \\
\hline 10 & High proportion of trials of this product are unpublished \\
\hline 11 & Postmarketing surveillance has identified safety concerns \\
\hline 12 & $\begin{array}{l}\text { Important or standard outcome measures (also known as 'end } \\
\text { points') have not been published }\end{array}$ \\
\hline 13 & $\begin{array}{l}\text { Concerns regarding a lack of published data on potential } \\
\text { harms of the product }\end{array}$ \\
\hline 14 & $\begin{array}{l}\text { Marketing authorisation based on surrogate outcomes (rather } \\
\text { than clinical outcomes) }\end{array}$ \\
\hline 15 & When protocol(s) are publicly available \\
\hline 16 & When statistical analysis plan(s) publicly available \\
\hline 17 & $\begin{array}{l}\text { Known errors or concerns about trial publications of this } \\
\text { product }\end{array}$ \\
\hline 18 & $\begin{array}{l}\text { Important discrepancies between the journal publication and } \\
\text { the trial registry entry }\end{array}$ \\
\hline
\end{tabular}

\section{The circumstances under which CSRs and/or other regulatory documents should be considered for inclusion in systematic reviews}

We concluded from the survey findings that the systematic review community is ready to consider using data from CSRs and other regulatory documents within systematic reviews. However, owing to the additional time and resource requirements that may be required to use these data sources, use should be focused on review topics where the data are needed most. We were unable to identify any research on the topic of how to decide whether to incorporate CSRs and other regulatory documents into systematic reviews, that is, a rule for determining which reviews would most benefit from the inclusion of such data.

We therefore created an initial list of reasons (or triggers) for seeking and using such data through discussion among our author group. Our list was a product of our opinion and experience. We then carried out a follow-up targeted survey in which we asked respondents to rate the importance of each criterion in our list. This survey was sent to the 21 (of 27) systematic review authors who had used, requested or considered using regulatory data in their review and had agreed to participate in a follow-up survey. Fourteen of $21(66 \%)$ provided a response. ${ }^{27}$

Table 2 shows our final list of criteria (after addressing review authors feedback) for assessing whether to include regulatory data of a drug or biologic in a systematic review. The variables are self-explanatory, reflecting either known or suspected bias in published results or the potential for greatest impact in terms of public health, for example, what are the human costs of acting on biased estimates of effectiveness or harm?

There is no proposed scoring or algorithm for combining criteria to identify priority topics or topic areas. The relative importance of criteria listed in table 2 will depend very much on context, and prioritisation is inevitably a somewhat subjective process. The list is not meant to be onerous. Systematic and formal evaluation of each of the 18 criteria is not required. We suggest reviewers instead focus on the items in the list that are most relevant to their research area of interest. The list is subject to revision and may even become obsolete over time as we learn more about the added value of using CSRs for systematic review, and if the ease of accessing CSRs becomes less problematic and burdensome in the future.

\section{Limitations when using CSRs}

There are potential limitations when using CSRs as the data source for systematic reviews. First, CSRs are written for regulators and may contain sensitive information that needs to be redacted. Redactions may delay the time it takes to obtain CSRs and the applied redactions may be extensive, masking important information for inclusion in systematic reviews. Second, although data on adverse events are required to be included in CSRs at the individual participant level, these data may not be fully provided to regulators (although regulators can request these data) or to systematic reviewers who request CSRs directly from manufacturers. For example, completed case report forms are not always held by the EMA and serious adverse event narratives are redacted from CSRs on GSK's clinical trial register. Third, complete CSRs may not be held by the EMA, and some CSRs may not be held at all, although a complete list of all clinical trials conducted by a manufacturer does form part of the regulatory submission. Fourth, a CSR is a compiled report of a study and not the study's underlying raw data. Despite all their strengths as a rich source of data, some investigators have identified deficiencies in the reporting in CSRs, specifically the completeness and consistency of reporting of adverse events. ${ }^{28-31}$

\section{Conclusion}

Regulatory documents are a complex and underused source of highly detailed data that could be included in systematic reviews. Although the steps to identify and extract and analyse data are broadly the same as for other sources of data, the resource implications of their use may not be. The results of our surveys and our own experience indicate that the use of regulatory documents should be considered, especially when the intervention in question is of high importance and when risk of reporting bias is great.

\section{Author affiliations}

${ }^{1}$ Centre for Evidence Based Medicine, Nuffield Department of Primary Care Health Sciences, Primary Sciences Division, University of Oxford, Oxford, UK

${ }^{2}$ Acute Respiratory Infections Group, Cochrane

${ }^{3}$ Department of Pharmaceutical Health Services Research, University of Maryland School of Pharmacy, Baltimore, Maryland, USA

${ }^{4}$ METHODs team, Centre of Research in Epidemiology and Statistics Sorbonne Paris Cité, INSERM UMR 1153, University Paris Descartes, Paris, France

${ }^{5}$ Risk of Bias Methods group, Cochrane

${ }^{6}$ Department of Health Sciences, University of York, York, UK

${ }^{7}$ Adverse Effects Methods Group, Cochrane

${ }^{8}$ Centre for Primary Care, Division of Population Health, Health Services Research \& Primary Care, University of Manchester, Manchester, UK

${ }^{9}$ Centre for Reviews and Dissemination, University of York, York, UK 
${ }^{10}$ School of Public Health, University of Queensland, Brisbane, Queensland, Australia

${ }^{11}$ Lefebvre Associates Ltd, Oxford, UK

${ }^{12}$ Information Retrieval Methods Group, Cochrane

${ }^{13}$ IPD meta-analysis group, Cochrane

Acknowledgements The authors are grateful for helpful comments on an earlier version of the draft from the following: Rene Allard, Chris Champion, Kay Dickersin, Kristina Charlotte Dietz, Jean-Mac Ferran, Mark Helfand, Toby Lasserson, Sherry Meeh, Kim Musgrave, Sarah Nevitt, Erick Turner and Beate Wieseler. The authors are very grateful to those who responded to calls for feedback and the authors endeavoured to incorporate their comments. Incorporation of feedback comments and/or listing of names above does not infer respondents' endorsement of this work.

Contributors All authors contributed to the research reported and the writing of the article.

Funding This research was supported by funds provided by Cochrane Methods Innovation Fund.

Disclaimer The views expressed in this final report are those of the authors and not necessarily those of Cochrane or its registered entities, committees or working groups.

Competing interests All authors except AH report funding from the Cochrane Methods Innovation Fund to undertake this project. In addition, TJ was a corecipient of a UK National Institute for Health Research grant (HTA-10/80/01 Update and amalgamation of two Cochrane reviews: neuraminidase inhibitors for preventing and treating influenza in healthy adults and children; https://www.journalslibrary.nihr.ac.uk/programmes/ $\mathrm{hta} / 108001 \# /$ ). TJ is occasionally interviewed by market research companies about phase I or II pharmaceutical products. In 20112014, TJ acted as an expert witness in a litigation case related to the antiviral oseltamivir, in two litigation cases on potential vaccine-related damage and in a labour case on influenza vaccines in healthcare workers in Canada. He has acted as a consultant for Roche (1997-1999), GSK (2001-2002), SanofiSynthelabo (2003) and IMS Health (2013). In 2014-2016, TJ was a member of three advisory boards for Boehringer Ingelheim. TJ was a member of an independent data monitoring committee for a Sanofi Pasteur clinical trial on an influenza vaccine. TJ has a potential financial conflict of interest on the drug oseltamivir. TJ was a cosignatory of a complaint to the European Ombudsman on maladministration in relation to the EMA investigation of possible harms from HPV vaccines. PD reports reimbursement from the European Respiratory Society, grants from UK NIHR, the American Association of Colleges of Pharmacy and the Laura and John Arnold Foundation, all outside the submitted work. PD is also an associate editor of The BMJ and an unpaid member of the IMEDS steering committee at the Reagan-Udall Foundation for the FDA, which focuses on drug safety research. IB is deputy director of the French EQUATOR centre; and member of the CONSORT steering committee. $\mathrm{CH}$ reports he has received expenses and fees for his media work. He has received expenses from WHO and holds grant funding from the NIHR, the NIHR School of Primary Care Research, THE NIHR BRC Oxford and WHO. He has received financial remuneration from an asbestos case. He has also received income from the publication of a series of toolkit books published by Blackwells. On occasion, he receives expenses for teaching EBM and is also paid for his GP work in NHS out of hours. CEBM jointly runs the Evidence Live
Conference with The BMJ and the Overdiagnosis Conference with some international partners, which are based on a nonprofit making model. He is Editor-in-Chief of BMJ EvidenceBased Medicine. LAS is an employee of the University of York. She previously received a research grant from YODA for her research team to independently analyse individual participant data and data from CSRs supplied by Medtronic.

Patient consent Not required.

Provenance and peer review Not commissioned; externally peer reviewed.

\section{References}

1. Dickersin K, Min YI. Publication bias: the problem that won't go away. Ann N Y Acad Sci 1993;703:135-48.

2. Dwan K, Altman DG, Arnaiz JA, et al. Systematic review of the empirical evidence of study publication bias and outcome reporting bias. PLoS One 2008;3:e3081.

3. Hopewell S, Loudon K, Clarke MJ, et al. Publication bias in clinical trials due to statistical significance or direction of trial results. Cochrane Database Syst Rev 2009;1:MR000006.

4. Ross JS, Tse T, Zarin DA, et al. Publication of NIH funded trials registered in ClinicalTrials.gov: cross sectional analysis. BMJ 2011;344:d7292.

5. Vedula SS, Bero L, Scherer RW, et al. Outcome reporting in industrysponsored trials of gabapentin for off-label use. $N$ Engl J Med 2009;361:1963-71.

6. McGauran N, Wieseler B, Kreis J, et al. Reporting bias in medical research a narrative review. Trials 2010;11:37.

7. Golder S, Loke YK, Wright K, et al. Most systematic reviews of adverse effects did not include unpublished data. J Clin Epidemiol 2016;77:125-33.

8. Schroll JB, Bero L, Gøtzsche PC. Searching for unpublished data for Cochrane reviews: cross sectional study. BMJ 2013;346:f2231.

9. Doshi P, Jefferson T. Clinical study reports of randomised controlled trials: an exploratory review of previously confidential industry reports. BMJ Open 2013;3:e002496.

10. Maund E, Tendal B, Hróbjartsson A, et al. Benefits and harms in clinical trials of duloxetine for treatment of major depressive disorder: comparison of clinical study reports, trial registries, and publications. BMJ 2014;348:g3510.

11. European Medicines Agency, 2017. Clinical data publication. http://www. ema.europa.eu/ema/?curl=pages/special_topics/general/ general_content_ 000555.jsp (cited 2 Oct 2017).

12. European Medicines Agency. European medicines agency policy on publication of clinical data for medicinal products for human use (POLICY/0070; EMA/240810/2013), 2014.

13. Health Canada, 2017. Public Release of Clinical Information in Drug Submissions and Medical Device Applications. https://www.canada.ca/ en/health-canada/programs/public-release-clinical-information-drugsubmissions-medical-device-applications.html (cited 2018 Feb 8).

14. Food and Drug Administration, 2018. Clinical data summary pilot program. http://www.fda.gov/Drugs/DevelopmentApprovalProcess/ucm589210.htm

15. Wieseler B, Kerekes MF, Vervoelgyi V, et al. Impact of document type on reporting quality of clinical drug trials: a comparison of registry reports, clinical study reports, and journal publications. BMJ 2012;344:d8141.

16. Anderson ML, Chiswell K, Peterson ED, et al. Compliance with results reporting at ClinicalTrials.gov. N Engl J Med 2015;372:1031-9.

17. National Institutes of Health, U.S. Department of Health and Human Services, 2016. NIH Policy on dissemination of NIH-funded clinical trial information. NOT-OD-16-149. https://grants.nih.gov/grants/guide/noticefiles/NOT-OD-16-149.html

18. Ross JS, Tse T, Zarin DA, et al. Publication of NIH funded trials registered in ClinicalTrials.gov: cross sectional analysis. BMJ 2012;344:d7292.

19. Schulz KF, Altman DG, Moher D. CONSORT 2010 Statement: updated guidelines for reporting parallel group randomised trials. BMC Med 2010;8:18.

20. Hopewell S, Dutton S, Yu LM, L-m Y, et al. The quality of reports of randomised trials in 2000 and 2006: comparative study of articles indexed in PubMed. BMJ 2010;340:c723. 
21. Plint AC, Moher D, Morrison A, et al. Does the CONSORT checklist improve the quality of reports of randomised controlled trials? A systematic review. Med J Aust 2006;185:263-7.

22. Loder E, Groves T. The BMJ requires data sharing on request for all trials. BMJ 2015;350:h2373.

23. Jefferson T, Jones MA, Doshi P, et al. Neuraminidase inhibitors for preventing and treating influenza in healthy adults and children. Cochrane Database Syst Rev 2014;4:CD008965.

24. Rodgers MA, Brown JV, Heirs MK, et al. Reporting of industry funded study outcome data: comparison of confidential and published data on the safety and effectiveness of rhBMP-2 for spinal fusion. $B M J$ 2013;346:f3981.

25. Fu R, Selph S, McDonagh M, et al. Effectiveness and harms of recombinant human bone morphogenetic protein-2 in spine fusion: a systematic review and meta-analysis. Ann Intern Med 2013;158:890-902.

26. Eyding D, Lelgemann M, Grouven U, et al. Reboxetine for acute treatment of major depression: systematic review and meta-analysis of published and unpublished placebo and selective serotonin reuptake inhibitor controlled trials. BMJ 2010;341:c4737.

27. Hodkinson A, Dietz KC, Lefebvre C, et al. The use of clinical study reports to enhance the quality of systematic reviews: a survey of systematic review authors. Syst Rev 2018;7:117.

28. Sharma T, Guski LS, Freund N, et al. Suicidality and aggression during antidepressant treatment: systematic review and meta-analyses based on clinical study reports. BMJ 2016;352:i65.

29. Le Noury J, Nardo JM, Healy D, et al. Restoring Study 329: efficacy and harms of paroxetine and imipramine in treatment of major depression in adolescence. BMJ 2015;351:h4320.

30. Hodkinson A, Gamble C, Smith CT. Reporting of harms outcomes: a comparison of journal publications with unpublished clinical study reports of orlistat trials. Trials 2016;17:207.

31. Vedula SS, Li T, Dickersin K. Differences in reporting of analyses in internal company documents versus published trial reports: comparisons in industry-sponsored trials in off-label uses of gabapentin. PLoS Med 2013;10:e1001378.
32. Chan AW, Hróbjartsson A, Haahr MT, et al. Empirical evidence for selective reporting of outcomes in randomized trials: comparison of protocols to published articles. JAMA 2004;291:2457-65.

33. Turner EH, Matthews AM, Linardatos E, et al. Selective publication of antidepressant trials and its influence on apparent efficacy. $N$ Engl J Med 2008;358:252-60.

34. Jefferson T, Jones MA, Doshi P, et al. Neuraminidase inhibitors for preventing and treating influenza in healthy adults and children. Cochrane Database Syst Rev 2012;1:CD008965.

35. Coyne DW. The health-related quality of life was not improved by targeting higher hemoglobin in the Normal Hematocrit Trial. Kidney Int 2012;82:235-41.

36. Wieseler B, Wolfram N, McGauran N, et al. Completeness of reporting of patient-relevant clinical trial outcomes: comparison of unpublished clinical study reports with publicly available data. PLoS Med 2013;10:e1001526.

37. Köhler M, Haag S, Biester K, et al. Information on new drugs at market entry: retrospective analysis of health technology assessment reports versus regulatory reports, journal publications, and registry reports. $B M J$ 2015;350:h796.

38. Beaumier J, Lawrence K, Puil L, et al. Olanzapine for schizophrenia: what do the unpublished clinical trials reveal? 23rd Cochrane Colloquium. Vienna, Austria, 2015.

39. Cosgrove L, Vannoy S, Mintzes B, et al. Under the influence: the interplay among industry, publishing, and drug regulation. Account Res 2016;23:257-79.

40. Jureidini JN, Amsterdam JD, McHenry LB. The citalopram CIT-MD-18 pediatric depression trial: Deconstruction of medical ghostwriting, data mischaracterisation and academic malfeasance. Int J Risk Saf Med 2016;28:33-43.

41. Schroll JB, Penninga EI, Gøtzsche PC. Assessment of adverse events in protocols, clinical study reports, and published papers of trials of orlistat: a document analysis. PLoS Med 2016;13:e1002101.

42. Mayo-Wilson E, Li T, Fusco N, et al. Cherry-picking by trialists and meta-analysts can drive conclusions about intervention efficacy. J Clin Epidemiol 2017;91:95-110. 